\title{
Design of smart printing System based on cloud computing
}

\author{
Haizhong Qian ${ }^{1, \text { a }}$, Lili Cai ${ }^{2, \mathrm{~b}}$ and Chishe Wang ${ }^{3, \mathrm{c}}$ \\ ${ }^{1}$ Jinling Institute of technology, Nanjing, Jiangsu,China \\ ${ }^{2}$ School of media technology, Communication university of China, Nanjing, \\ China \\ ${ }^{3}$ Jinling Institute of technology, Nanjing, Jiangsu,China \\ ahzhqian913@gmail.com, ${ }^{b}$ lilicai710@163.com, ${ }^{c}$ wangcs@jit.edu.cn
}

\begin{abstract}
.
Much applications based on cloud computing has emerged. This cloud print, such as HP ePrint, Google print is one of the typical. Archiving the vision of cloud print must solve two key problems. One is supporting the variety of the printing format; the other is how to locate the print devices on the INTERNET. In this paper, it aims to solve the two key problems and design a smart print System on the INTERNET. The smart print system is being applied on the Youth Olympics Game in Nanjing on August 2014. It has achieved great effect and to be proofed. Keywords: cloud computing, printing System, smart print, Architecture, PCL
\end{abstract}

\section{Introduction}

With the rapid development of the Internet gave birth to the Cloud Computing $^{[1,2]}$, the emergence of the Cloud Computing model can make ordinary users enjoy high performance Computing resources, software resources, hardware resources and service resources. Cloud computing "cloud" is similar to today's Internet, which is composed of the hardware resources (such as network devices, storage devices, server cluster, hardware services, etc.) and software resources (such as operating systems, applications, integrated development environment, software and services). User need only a simple terminal through a communication network connected to the "cloud" and deal with all the works the same as do the traditional computer network. 
The printer is essential office equipment of enterprises and institutions on the 21st century, compared to traditional printing, cloud printing via corresponding software and hardware systems to work instead of the PC. Only when the printer is connected to the "cloud", the printer will be as a service. Everyone can use the service by Intelligent Terminal in anytime, and on anywhere. It will be to bring people convenience on the modern Internet technology. Cloud Print ${ }^{[3,4]}$ will become an important model for future Printing Industry.

\section{Architectures of Smart Print System}

\section{A. overview}

Cloud Print system is built based on B / S mode. Everyone can use their intelligent terminals ${ }^{[5]}$ (such as: tablet computers, smart phones, computers, etc.) to access web server of Cloud Print system. If the user is certified, he (she) can use the cloud print service. Firstly, the file to be printed, which will be to upload to the print System; and then the user can select the wanted online printer; finally the file will be received the corresponded printer and to be printed.

Design the smart print system is based the topology as show Fig1.

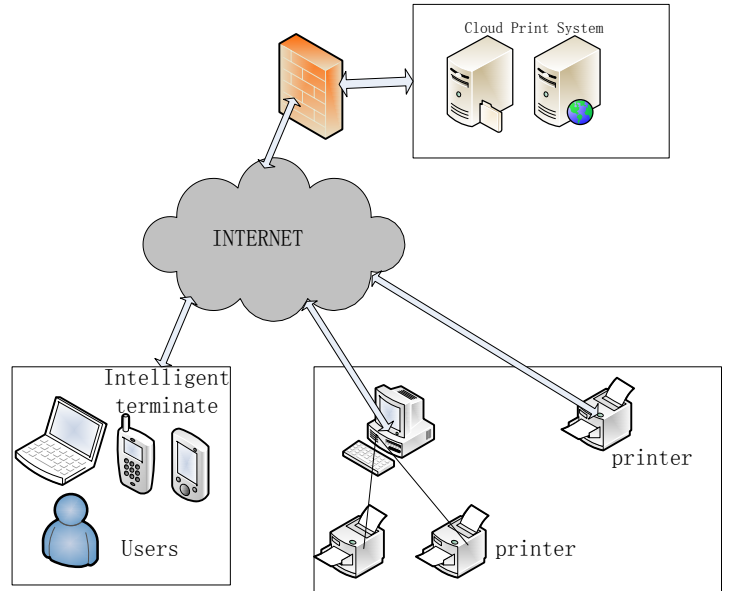

Fig1: the topology of smart print System 


\section{B. Support the variety of the printing format}

Due to several factors: print device have various brands on the presence market, they are supported by the printer language also has the big difference; cloud print but also support a variety of file formats; customers do not need to install the printer driver. Therefore, the cloud print System needs to implement the conversion of the variety of file format. The proccess of file format conversion as show Fig2.

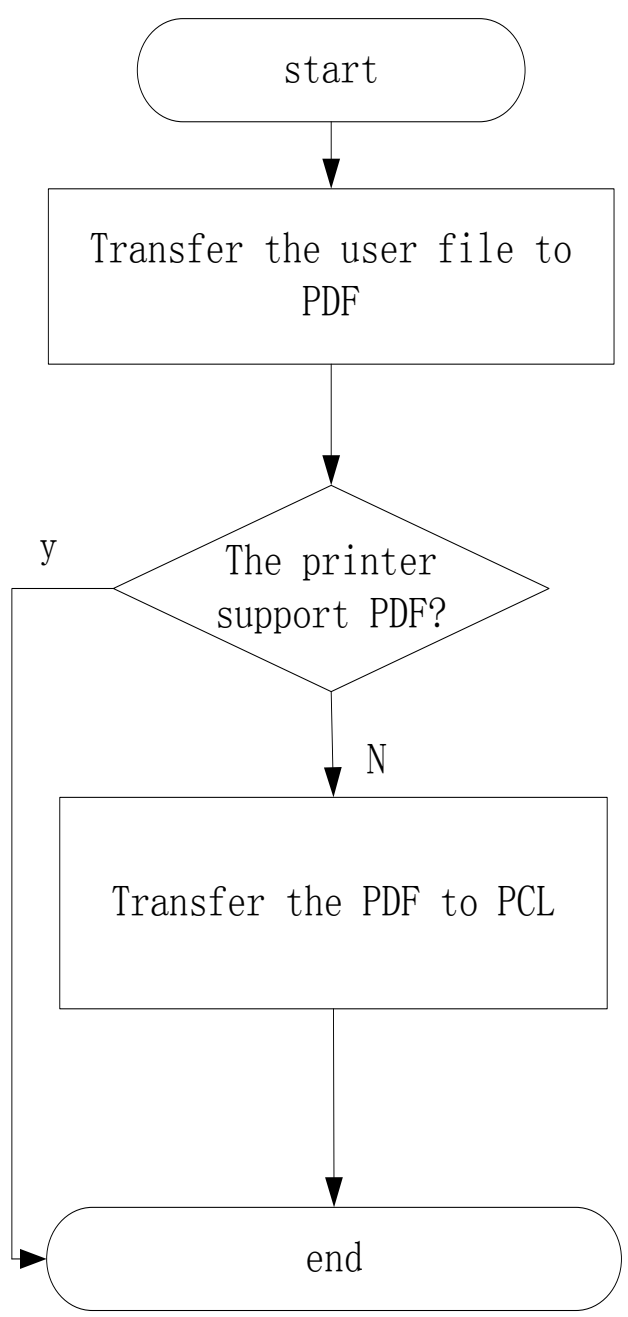

Fig2: The proccess of file format conversion 
Firstly, In addition to PDF file format, regardless of what kind of user-submitted format files are converted into PDF format. And then if the printer supported the PDF file format, this file can't be transferred to PCL file format by calling the "print to file" function of printer driver, otherwise the file will be transfer to PCL format before sending to printer.

\section{Locating the printer Device}

Print service ${ }^{[6,7]}$ is to be implemented eventually by the specific printer device. Due to the following factors: the printer may be located in a private address's network and printer without network connectivity, how to make these printers are able to facilitate sharing convenient in cloud printing system is one of the key problems of the cloud print. This paper designs a print agent module in order to solve the above problem.

Print agent has the some functions as follow: one is network communication, which can access the Internet and can be connected to the printer; second is communicating with the printer, which can call the function of printer, sent printing file to the printer task queue and obtain various state of printer. Print agent as a software module can be embedded into the printer device, can also be installed in a desktop computer, notebook computer, tablet computer and so on a series of intelligent terminal.

All printers are required to be registered to the print server by their print agent. The interaction diagram of the print agent, print servers and printers is as shown Fig3. 


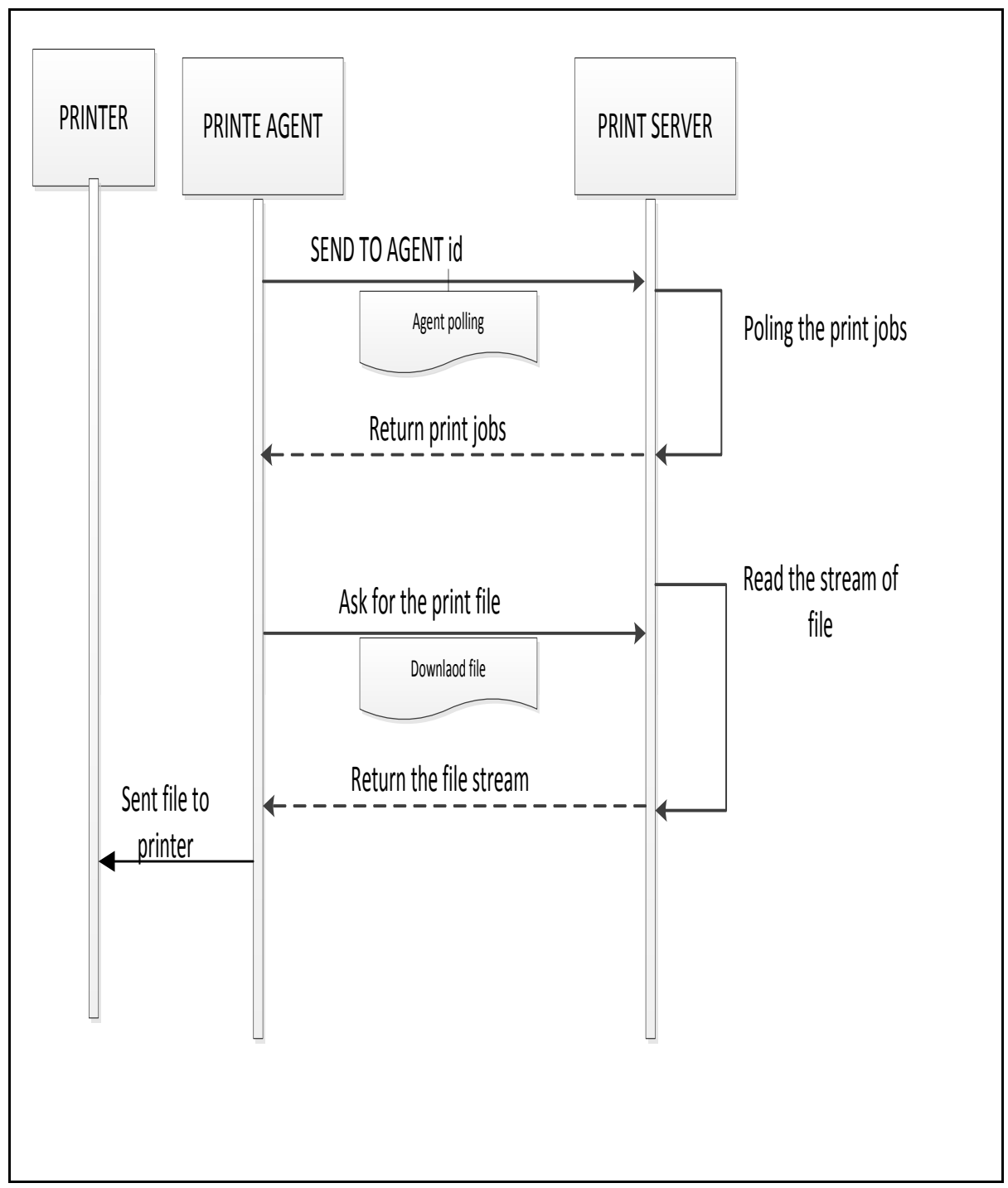

Fig3: The interaction diagram of the print agent, print servers and printers

Print Agent will connect the print server at each interval time and ask for the print job. The print server can obtain the online status of the printer. 
When the print agent has some print jobs, it will download these print jobs to local and call the printer to execute the printing tasks. The status of the print jobs also will be reported to the print Server when the printer finished printing tasks.

\section{Demonstration of the smart print system}

The Second Youth Olympic Games (YOG) will be held in Nanjing in 2014, China. Cloud Print aim is to provide the convenient printing services for athletes, volunteers and workers during this period。 It also will be able to play printing devices for maximum efficiency and reduce the count of printing devices. Cloud Print is once an important demonstration of the system in YOG.

Cloud printing system obtains outstanding performance during the period Of YOG, which will be provided the valuable practical value when the system is applied to printing industry. For the detail of the print system, you can see http://cloud.jit.edu.cn.

\section{Conclusion}

Cloud print is essentially the use of cloud computing technology to realize network existing print, precisely, is the era of mobile Internet network printing. This paper discusses the design of cloud print service platform and look at the many new technologies such as: cloud computing, mobile Internet, cloud services. It also aims to establish the new ecosystem of the printing industry and up grading the traditional printing industry.

\section{Acknowledgement}

This works is supported by “The Program of Jiangsu Province Science and Technology Department of Science and Technology”, No.BE2012604. 


\section{References}

[1] J. Baliga, R. W. A. Ayre,; K. Hinton; etc; Green Cloud Computing: Balancing Energy in Processing, Storage, and Transport, Proceedings of IEEE,Vol.99,No.1 p. 149-167, 2011.

[2] K. M., Sim; Agent-Based Cloud Computing, IEEE Transactions on Services Computing, Vol.5,No.4, p.564-577, 2011.

[3]Y.,Zhu; W.,Wu; L. Wang; etc; SmartPrint: A Cloud Print System for Office, 2013 IEEE Ninth International Conference on 2013 IEEE Ninth International Conference on Mobile Ad-hoc and Sensor Networks (MSN), p.95-100, 2013.

[4]Y.,Wu; Research of Cloud Print Key Technology Based on Identity Card , 2012 Third World Congress on Software Engineering (WCSE), p.176-178, 2012.

[5] Leeladevi, B. ; Rahul Raj, C.P. ; Tolety, S.; A study on smartphone printing approaches[C] ;2013 IEEE Conference on Information \& Communication Technologies (ICT), 2013,p 707-711.

[6] Zeng, Jun ; Jun Li ; Operations simulation as a cloud based service; IEEE Conference Anthology, 2013,p1-5.

[7] Hyuck Han ; Shingyu Kim ; Hyungsoo Jung; A RESTful Approach to the Management of Cloud Infrastructure ; IEEE International Conference on Cloud Computing; 2009 ,p139-142 